\title{
Serum Immunoglobulin G Levels in Small-for-Dates Newborn Babies
}

\author{
C. PAPADATOS, G. J. PAPAEVANGELOU, D. ALEXIOU, and J. MENDRIS \\ From the Neonatal Department of Alexandra Maternity Hospital and the Department of Hygiene and Epidemiology, \\ University of Athens, Athens, Greece
}

Papadatos, C., Papaevangelou, G. J., Alexiou, D., and Mendris, J. (1970). Archives of Disease in Childhood, 45, 570. Serum immunoglobulin G levels in small-for-dates newborn babies. Immunoglobulin $G$ levels have been studied in 47 small-for-dates (SFD) and 197 singieton babies of normal birthweight for their gestational age.

The IgG values in the SFD babies were below those expected for the normal newborn of an equivalent gestational age, but above those of the normally growing baby of equivalent birthweight. There is a statistically significant difference in the slopes of the regression lines in the two groups whether IgG is plotted against birthweight or gestational age.

Under normal conditions the human fetus produces immunoglobulins in minute amounts (Stiehm and Fudenberg, 1966; Johansson and Berg, 1967). However, levels of IgG at birth approach and even surpass adult serum concentrations; nearly all this IgG in the newborn is of maternal origin (Bridges et al., 1959).

Low concentrations of IgG have been reported in obviously premature infants (Hobbs and Davis, 1967; Papadatos et al., 1969). With advancing gestational age these levels tend to rise and a linear relation has been shown to exist between IgG concentration and gestational age (Yeung and Hobbs, 1968; Papadatos et al., 1969).

While findings in post-mature babies are contradictory (Yeung and Hobbs, 1968; Papadatos et al., 1969; Ackerman, Taylor, and O'Loughlin, 1969), results in small-for-dates (SFD) newborn babies, though based on a small number of observations (Yeung and Hobbs, 1968), show that IgG levels remain below the corresponding mean values for their gestational age.

In this report we provide additional information on immunoglobulin $G$ in the SFD baby.

\section{Material and Methods}

Our material consisted of 197 normal singleton newborns, with a gestational age of 24 to 48 weeks, and 47

Received 25 January 1970.
SFD babies with a gestational age of 36 to 44 weeks, born at the Alexandra Maternity Hospital from January 1968 to July 1969 . Newborns with congenital malformations were excluded from the study. Their gestational age was estimated from the first day of the mother's last menstrual period, and was considered accurate if the mother stated that she was sure of her dates. An attempt was made to correlate the gestational age to neurological examination. Specific tests that have been described by different authors (Brett, 1965; Farr, 1968; Koenigsberger, 1966; Robinson, 1966; Saint-Anne-Dargassies, 1955) as well as morphological criteria described by Usher, McLean, and Scott (1966) and Farr et al. (1966) have been used in all our babies.

The SFD group had birthweights 2 SD or more below the mean for their gestational age according to the developmental curves of the British Perinatal Mortality Survey (Butler and Alberman, 1969).

The umbilical cords were clamped immediatelywithin 10 seconds after delivery. Placental studies were not done. Blood samples were obtained by venepuncture under aseptic conditions within the first 24 hours after birth, and the serum was maintained after separation at $-20^{\circ} \mathrm{C}$. The determination of all serum IgG levels was done by using the quantitative gel diffusion technique with antibody incorporated into the agar in appropriate dilution as described by Mancini, Carbonara, and Heremans (1965).

\section{Results}

As previously shown, a linear relation exists between serum $\log \mathrm{IgG}(\mathrm{mg} . / 100 \mathrm{ml}$.) levels and gestational age from the 24th up to the 42 nd week 


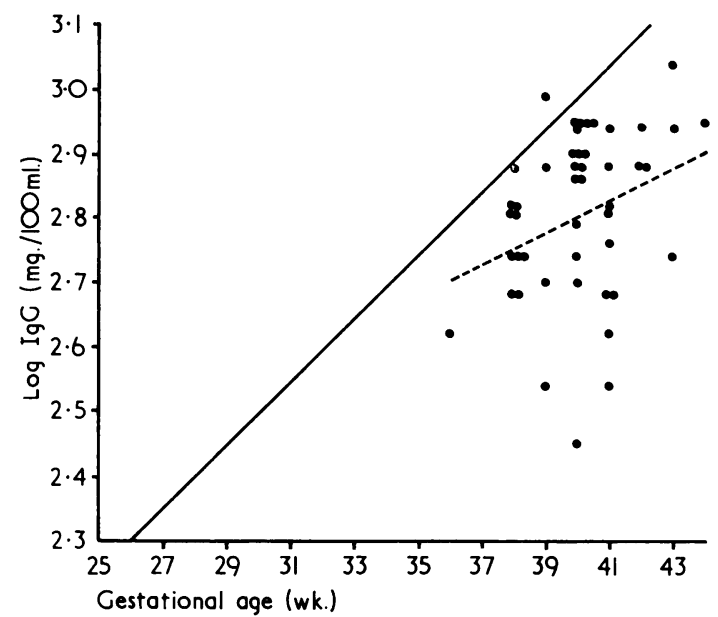

FIG. 1.-Correlation of log serum IgG levels to gestational age (weeks) in normal newborns (- $\longrightarrow$ ) and small-fordates (-----) babies. The circles ( $\bullet$ ) represent the individual values of IgG in the SFD babies.

(Yeung and Hobbs, 1968; Papadatos et al., 1969). Complete data, including $95 \%$ confidence limits on the 197 normal newborns have already been published (Papadatos et al., 1969). The best fitting regression line for mean IgG values at each gestational age is shown in Fig. 1. All except one of the 47 SFD babies had IgG levels below the mean for their gestational age.

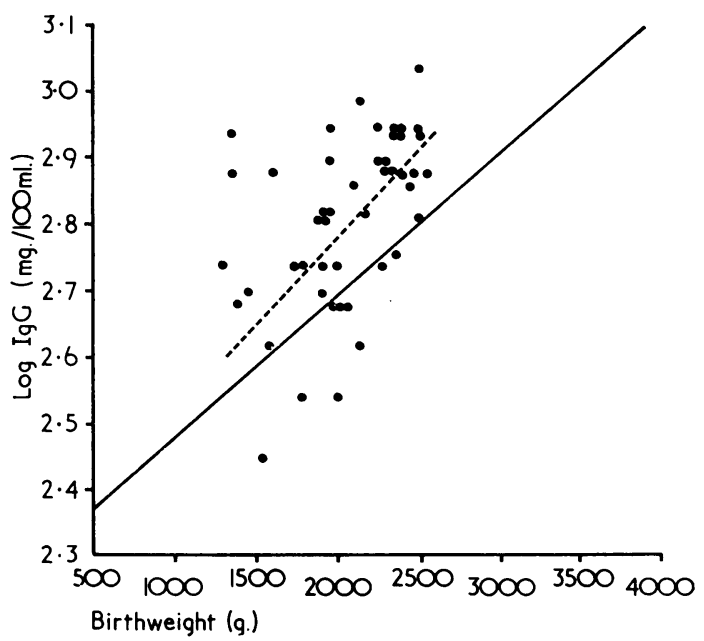

FIG. 2.-Correlation of log IgG level to birthweight (g.) in normal newborns (- $\longrightarrow$ ) and small-for-dates (-------) babies. The circles $(\bullet)$ represent the individual values of Ig $G$ in SFD babies.
In the study of the relation between length of pregnancy and IgG concentration, gestational age may be difficult to estimate in some individual cases. We approached the problem by trying to correlate the date of the first day of the last menstrual period of the mother with the neurological evaluation of the newborn as described by different authors (Brett, 1965; Farr, 1968; Robinson, 1966; Koenigsberger, 1966; Saint-Anne-Dargassies, 1955) and the morphological criteria described by Usher et al. (1966) and Farr et al. (1966). We found the babies' responses very variable, and did not feel confident in the use of any of the proposed methods. We therefore relied on maternal information.

Further statistical analysis of our results shows that the $\log$ IgG levels in the SFD babies are directly correlated to their gestational age. The correlation coefficient is found to be $0.31(0.01<\mathrm{p}$ $<0.05)$. The best fitting regression line of $\mathrm{Y}$ on $\mathrm{X}$ is shown in Fig. 1 (dashed line) and its equation is: $\log \operatorname{IgG}=1.8043+0.0250 \times$ gestation in weeks.

Though the number of SFD babies studied is small, the analysis of variance appears to justify the assumption that the relation is linear $(F=0.99$, $\mathrm{p}<0 \cdot 05)$. The comparison of the two regression lines shows that their slopes differ significantly $(t=9 \cdot 45, p<0 \cdot 001)$.

Fig. 2 shows the correlation between $\log$ IgG levels and birthweight. We used logarithmic rather than arithmetic values of IgG levels because the $\log$ values gave a better fit to a straight line relation. In the 197 babies of normal birthweight for their gestational age, the correlation coefficient was $0.78(\mathrm{p}<0.01)$ and the equation for the regression line through the means was:

$\log \mathrm{IgG}=0 \cdot 000215 \times$ birthweight in g. $+2 \cdot 26478$.

At a given birthweight the SFD babies had slightly higher IgG levels than the babies of normal birthweight for their gestation (Fig. 2). Only 9 of them $(19 \%)$ had IgG values below the corresponding mean for normal newborns of equivalent birthweight. Our results on the SFD babies also show that there is a smaller $(r=0.62)$ but significant $(p<0.01)$ correlation. The regression line (dashed) is shown in Fig. 2. The slopes of the two regression lines differ significantly $(t=3.9 ; p<0 \cdot 05)$.

\section{Discussion}

The few previous studies on IgG levels in SFD babies have shown values below the mean for the corresponding gestational age (Yeung and Hobbs, 1968; Papadatos et al., 1969). It should be stressed, however, that SFD babies do not represent a homogeneous population (Wigglesworth, 1967), for 
limitation of fetal growth support, loosely considered as 'placental' insufficiency, has not been fully elucidated.

In the present study we compared IgG levels in two groups of babies. The first included 197 newborns with a birthweight within the mean $\pm 2 \mathrm{SD}$ for their gestational age, while in the second group we studied 47 SFD babies. A dependence of IgG levels on gestational age was found in both groups. With one exception SFD babies had levels below the mean for their gestational age. The regression coefficient in the two groups measures the average amount by which IgG increases for each week's increase in gestational age. The statistically significant difference between the two regression coefficients denotes that this increase is slower in SFD newborns, which is in good agreement with the general pattern of growth in these babies.

The IgG level of the newborn is not the result of a passive but rather of an active placental transport from mother to fetus. It is reasonable to suppose that the relatively slow increase of IgG in the SFD fetus, like the slow fetal growth, indicates inadequate placental function.

Our results show that for the normally growing babies there is also a significant correlation between birthweight and IgG. In the SFD newborns this correlation is smaller but nevertheless significant. In the SFD babies the average increase in IgG is significantly greater than in babies with normal growth, and therefore SFD babies have greater amounts of IgG than normally growing newborns with similar birthweights.

\section{REFERENCES}

Ackerman, B. D., Taylor, W. F., and O'Loughlin, B. J. (1969). Serum immunoglobulin levels in postmature infants. Pediatrics, 43, 956.
Brett, E. M. (1965). The estimation of foetal maturity by the neurological examination of the neonate. Clinics in Developmental Medicine, 19, 105.

Bridges, R. A., Condie, R. M., Zak, S. J., and Good, R. A. (1959). The morphologic basis of antibody formation development during the neonatal period. Fournal of Laboratory and Clinical Medicine, 153, 331 .

Butler, N. R., and Alberman, E. D. (1969). Perinatal Problems: The Second Report of the 1958 British Perinatal Mortality Survey. Livingstone, Edinburgh.

Farr, V. (1968). Estimation of gestational age by neurological assessment in first week of life. Archives of Disease in Childhood, 43, 353.

—, Mitchell, R. G., Neligan, G. A., and Parkin, J. M. (1966). The definition of some external characteristics used in the assessment of gestational age in the newborn infant. Developmental Medicine and Child Neurology, 8, 507.

Hobbs, J. R., and Davis, J. A. (1967). Serum- $\sigma$ G-globulin levels and gestational age in premature babies. Lancet, 1, 757.

Johansson, S. G. O., and Berg, T. (1967). Immunoglobulin levels in healthy children. Acta Paediatrica Scandinavica, 56, 572.

Koenigsberger, M. R. (1966). Judgment of fetal age. I. Neurologic evaluation. Pediatric Clincis of North America, 13, 823.

Mancini, G., Carbonara, A. O., and Heremans, J. F. (1965). Immunochemical quantitation of antigens by single radial immunodiffusion. Immunochemistry, 2, 235.

Papadatos, C., Papaevangelou, G., Alexiou, D., and Mendris, J. (1969). Immunoglobulin levels and gestational age. Biologia Neonatorum, 14, 365.

Robinson, R. J. (1966). Assessment of gestational age by neurological examination. Archives of Disease in Childhood, 41, 437.

Saint-Anne-Dargassies, S. (1955). La maturation neurologique du prématuré. Études Neo-natales, 4, 71.

Stiehm, E. R., and Fudenberg, H. H. (1966). Serum levels of immune globulins in health and disease: a survey. Pediatrics, 37, 715.

Usher, R., McLean, F., and Scott, K. E. (1966). Judgment of fetal age. II. Clinical significance of gestational age and an objective method for its assessment. Pediatric Clinics of North America, 13, 835.

Wigglesworth, J. S. (1967). Pathological and experimental aspects of foetal growth retardation. Proceedings of the Royal Society of Medicine, 60, 879.

Yeung, C. Y., and Hobbs, J. R. (1968). Serum- $\alpha$ G-globulin levels in normal, premature, post-mature and 'small-for-dates' newborn babies. Lancet, 1, 1167.

Correspondence to Dr. C. Papadatos, Alexandra Maternity Hospital, Neonatal Department, Athens (611), Greece. 